\title{
miR-216b Post-Transcriptionally Downregulates Oncogene KRAS and Inhibits Cell Proliferation and Invasion in Clear Cell Renal Cell Carcinoma
}

\author{
Yan Wang $\quad$ Daoquan Dong ${ }^{a} \quad$ Shuqi Jianga Enpu Zhang ${ }^{a} \quad$ Wenzhong Zheng ${ }^{a}$ \\ Longyi Mao ${ }^{c}$ Weiqing $\mathrm{Li}^{\mathrm{a}}$ Jingcheng Zhou ${ }^{\mathrm{a}}$ Longlong Fan ${ }^{\mathrm{a}}$ Ran Cheng ${ }^{\mathrm{a}}$ \\ Zesong $\mathrm{Li}^{\mathrm{c}}$ Zhengyu Fang ${ }^{\mathrm{a}}$ Yaoting Gui ${ }^{\mathrm{a}}$ Xianxin Lia,b \\ aDepartment of Urology, Peking University Shenzhen Hospital, Shenzhen PKU-HKUST Medical Center, \\ Shenzhen, bDepartment of Surgical, Shenzhen Sun Yat-sen Cardiovascular Hospital, Shenzhen, \\ 'Guangdong Key Laboratory of Systems Biology and Synthetic Biology for Urogenital Tumors, \\ Shenzhen Key Laboratory of Genitourinary Tumor, Shenzhen Second People's Hospital, First Affiliated \\ Hospital of Shenzhen University, Shenzhen, China
}

\section{Key Words}

MicroRNA-216b • KRAS • Renal cell carcinoma

\begin{abstract}
Background/Aims: Increasing evidence has shown that miR-216b plays an important role in human cancer progression. However, little is known about the function of miR-216b in renal cell carcinoma. Methods: The expression levels of miR-216b in renal cell carcinoma tissues and cell lines were examined by qRT-PCR. The biological role of miR-216b in renal cell carcinoma proliferation and/or metastasis was examined in vitro and in vivo. The target of miR-216b was identified by a dual-luciferase reporter assay. The expression level of KRAS protein was measured by western blotting. Results: The expression of miR-216b was downregulated in clear cell renal cell carcinoma (ccRCC) cell lines and specimens compared to the adjacent normal tissues. Furthermore, miR-216b can bind to the 3'untranslated region (UTR) of KRAS and inhibit the expression of KRAS through translational repression. The in vitro study revealed that miR-216b attenuated cCRCC cell proliferation and invasion. Furthermore, in vivo study also showed that miR-216b suppressed tumor growth. MiR-216b exerted its tumor suppressor function through inhibiting the KRAS-related MAPK/ERK and PI3K/AKT pathways. Conclusion: Our findings provide, for the first time, significant clues regarding the role of miR-216b as a tumor suppressor by targeting KRAS in cCRCC.




\section{Cellular Physiology Cell Physiol Biochem 2018;49:1755-1765

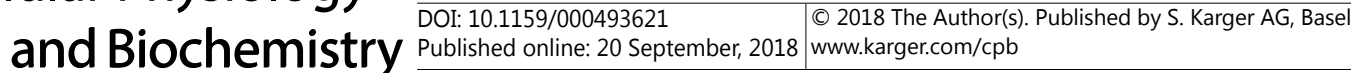 \\ Wang et al.: miR-216b, a Tumor Suppressor in cCRCC}

\section{Introduction}

Renal cell carcinoma (RCC) is the most common neoplasm of the kidney, accounting for $2-3 \%$ of all adult urologic malignancies. Among several histological subtypes, clear cell renal cell carcinoma (ccRCC) is the most frequent, presenting $70 \%-80 \%$ of RCC cases [1]. ccRCC is characterized with the frequent loss of $3 p$ [2], and several genes have been involved in its pathogenesis. In addition to protein coding genes, microRNAs (miRNAs) are involved in the pathogenesis of ccRCC. More and more miRNAs have been shown to exert tumor suppressive or oncogenic function in a tissue-specific manner [3-5]. Aberrant expression of these miRNAs results in differential translational control of numerous target genes. This can result in the deregulation of cellular processes like proliferation, differentiation, programmed cell death, and morphogenesis, thereby contributing to the process of tumor development [3-5]. Several groups have demonstrated up- and downregulated miRNAs in primary ccRCC tumors compared to normal kidney tissues [4, 6, 7]. Aberrant expression of miR-216b has been found in various cancers, including melanoma, cervical cancer, pancreatic cancer, breast cancer and hepatocellular carcinoma [8-12]. In our preliminary study, miRNA sequencing results showed that miR-216b was downregulated in ccRCC tissue compared to the adjacent normal tissue. However, the biological function and downstream targets of miR$216 \mathrm{~b}$ in ccRCC remain largely unknown.

Kirsten rat sarcoma viral oncogene homolog (KRAS) oncogene, one of the RAS gene family members, encodes a small guanosine triphosphatase, which has crucial functions in various biological processes, including cell proliferation and metastasis. Downregulation of KRAS has been shown to reduce tumor growth in pancreatic cancer, glioma, and oral cancer, among others. However, the role of KRAS in the progress of ccRCC is unknown. Recently, it is reported that there is no mutation in the known gene loci on exons 2 and 15 of KRAS in renal cell carcinoma $[13,14]$. Therefore, miR-216b and KRAS may be viable biomarkers or potential therapeutic targets for ccRCC.

Here, we determined that miR-216b was downregulated in ccRCC. MiR-216b directly targets the 3'-UTR of the KRAS transcript and elicits a specific and robust knockdown of KRAS protein, and it also inhibits ccRCC cell proliferation and invasion. MiR-216b mediates its tumor suppressor function, at least in part, by suppressing pathways downstream of KRAS, such as the PI3K-AKT and MEK-ERK pathways. Our findings provide, for the first time, significant clues regarding the role of miR-216b as a tumor suppressor in ccRCC.

\section{Materials and Methods}

Tissue Specimens and Cell culture

A total of 31 pairs of renal cell carcinoma tissues and related cancer-adjacent normal tissues were obtained from Shenzhen clinical biobank of major disease, China. All the tumor tissues confirmed by pathology were preserved in $-80^{\circ} \mathrm{C}$. This project was approved by the Ethical Committee of Shenzhen hospital, Peking University, China.

This study employed human ccRCC cell lines including ACHN, 786-0, 769-P, Caki-1 and Caki-2. All cell lines were obtained from the American Type Culture Collection (Manassas, VA, USA). ACHN cells were grown in Eagle's Minimum Essential Medium supplemented with $10 \%$ fetal bovine serum (FBS) and $1 \mathrm{~mm} / \mathrm{L}$ penicillin-streptomycin. Caki-1 and Caki-2 cells were cultured in McCoy's 5a Medium supplemented with $10 \%$ FBS and 1mm/L penicillin-streptomycin. 786-0 and 769-P cells were cultured in RPMI-1640 medium supplemented with $10 \% \mathrm{FBS}$ and $1 \mathrm{~mm} / \mathrm{L}$ penicillin-streptomycin. All cells were maintained at $37^{\circ} \mathrm{C}$ under an atmosphere of $5 \% \mathrm{CO}_{2}$ and $95 \%$ air.

MiRNA expression analysis

Total RNAs were extracted from cells with TRIzol reagent (Invitrogen). For miR-216b, reverse transcription and qRT-PCR reactions were performed by means of a qSYBR-green-containing PCR kit (Qiagen, USA), and U6 snRNA was used as an endogenous control for miRNA detection. The primers used in 


\section{Cellular Physiology Cell Physiol Biochem 2018;49:1755-1765

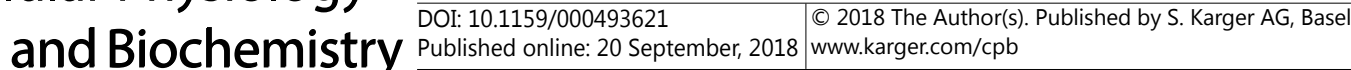 \\ Wang et al.: miR-216b, a Tumor Suppressor in ccRCC}

this study are shown in Table 1 . The fold change of gene expression from the different treatment as compared with control groups was calculated

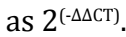

Construct of Luciferase reporter vectors and KRAS-expressing vector

The full-length KRAS 3'-UTR was cloned into the Sac I and Mlu I sites of the pSicheck-2 luciferase vector using the PCR generated fragment. The mutant vector pSiKRAS $_{\text {mut }}$ in which the first five nucleotides complementary to the miR-216b seed-region were mutated by site-directed mutagenesis, was constructed as a mutant control. Fulllength KRAS cDNA entirely without the 3'-UTR was purchased from Origen (Rockville, MD, USA) and subcloned into the eukaryotic expression vector pCMV-Entry vector. The primers used in this study are shown in Table 1 . The empty pCMV-Entry vector was used as a negative control.

\section{Cell transfection}

For the function experiments of miR-216b, the miR-216b mimics and the negative scramble control were transfected into 786-O and ACHN cells using Lipofectamine 3000 (Invitrogen). The inhibitors antimiR$216 \mathrm{~b}$ and anti-scramble were also transfected into 786-0 and ACHN cells. For miRNA and pCMV-KRAS combination experiments, 786-0 and ACHN cells were transfected with pCMV-KRAS or empty vector 24 hours together with miR-216b mimics or inhibitors.

\section{Dual Luciferase assay}

$\mathrm{pSi}^{-\mathrm{KRAS}_{\mathrm{wt}}}$ and pSi-KRAS ${ }_{\text {mut }}$ were co-transfected with miR-216b mimics into 786-0 cells. Luciferase activity was measured in cell lysates 48 hours after transfection using the Dual-luciferase reporter gene assay kit (Promega). Results were normalized against b-galactosidase activity.

\section{Protein Analysis}

Western blotting was carried out as described [15]. Anti-KRAS antibody was obtained from SigmaAldrich. Antibodies against ERK, phosphorylated ERK, AKT and phosphorylated AKT antibodies were obtained from Cell Signaling Technology. Anti- $\beta$-tubulin antibody was from abcam.

\section{Cell proliferation assay}

After transfection, cells were plated into 96-well plates at the desired cell concentrations. Cells were analyzed at 0, 24, 48 and 72 hours using the Cell Counting kit-8 (CCK-8) (Dojindo Laboratories, Kumamoto, Japan) with a Coulter counter (Beckman Coulter, Fullerton, CA) at the indicated time points.

\section{Cell invasion assay}

At 48 hours after transfection, cells were seeded onto the basement membrane matrix (EC matrix, Chemicon, Temecula, CA) present in the insert of a 24-well culture plate. Complete culture medium was added to the lower chamber as a chemoattractant. After a further 24 hours, the non-invading cells and EC matrix were gently removed with a cotton swab. Invasive cells located on the lower side of the chamber were fixed with $4 \%$ paraformaldehyde and stained with Crystal Violet, air-dried and photographed with microscope (Nikon Elipse Ci, Japan). 
In vivo animal model

For the subcutaneous tumor growth assay, $2 \times 10^{6}$ cells were suspended in $0.1 \mathrm{ml}$ of PBS and implanted subcutaneously into the flanks of six-week-old male balb/c nu-nu mice. Tumor growth was examined twice a week, and tumor volume was estimated by the formula $\mathrm{LW}^{2} / 2$, where $\mathrm{L}$ is the length and $\mathrm{W}$ is the width of the tumor. Tumor weight was measured at the end of the study. All animal procedures were performed with the approval of the Ethical Committee of Shenzhen hospital, Peking University, China.

\section{Statistical Analysis}

All experiments were performed in triplicate. The data were statistically evaluated by analysis (ANOVA) and comparison means (student's t-test). A $p$ value of less than 0.05 indicated the presence of statistically significant difference between groups. All statistical analyses were carried out with Graphpad Prism 6.

\section{Results}

\section{MiR-216b is downregulated in ccRCC cell lines and clinical specimens}

In our previous study, miR-216b was found to be downregulated in ccRCC specimens as assessed by microarray analysis [16]. Here we identified the expression of miR-216b both in 31 ccRCC and in specimens by qPCR. MiR-216b was reduced in clinical ccRCC specimens compared to the matched adjacent normal specimens (Fig. 1A and B, p<0.001).

Fig. 1. MiR-216b is downregulated in ccRCC tissues and cell lines. (A, B) qRTPCR analysis of the expression levels of miR-216 in 31 clinical ccRCC tissues (T) and matched adjacent normal tissues $(\mathrm{N})$. The expression values of one sample are set at 1 for miR$216 \mathrm{~b}$. The relative expression levels of mir-216b were determined by using the $2^{(-\Delta \Delta \mathrm{CT})}$ method. (C) qRT-PCR analysis of the expression levels of miR$216 \mathrm{~b}$ in five ccRCC cell lines. The relative expression level of miR-216b was determined by using the $2^{(-\Delta \Delta C T)}$ method. (D) Protein analysis of KRAS in ten pairs of clinical clear cell renal cell carcinoma specimens and adjacent specimens. KRAS was dramatically upregulated in six ccRCC tissues. (E and F) Efficacy of has-miR-216b mimics in ccRCC cells. Transfection with miR-216b mimics increased miR-216b expression in 786$O$ and ACHN cells. Mean \pm SEM, t-test, ${ }^{* * *} \mathrm{p}<0.001$.
A

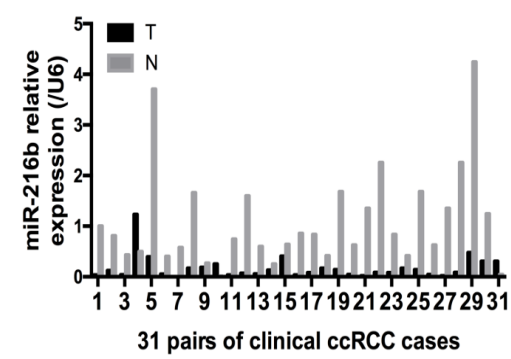

C

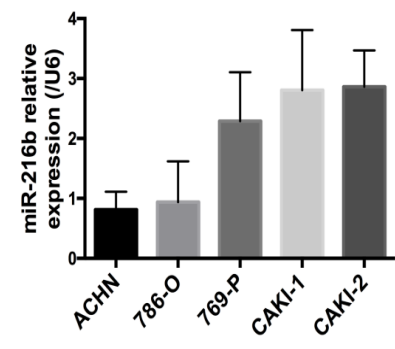

E

D

\section{B}

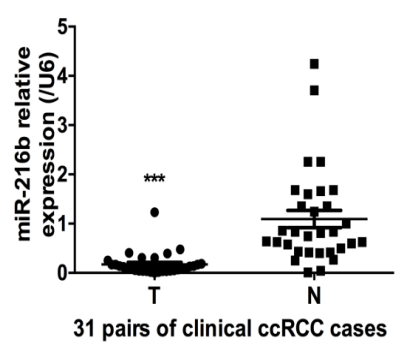


To study the role of miRNA in the progress of ccRCC, we analyzed the different expression of miR-216b in five ccRCC cell lines by qPCR. The lower expression of miR-216b was found in 786-0 and ACHN cell lines compared to that in other three cell lines: namely 769-P, Caki1 and Caki-2 (Fig. 1C). Therefore, 786-O and ACHN were selected for subsequent function studies.

To investigate the role of miR-216b, we transfected miR-216b mimics or inhibitors in 786-O and ACHN to increase or decrease the expression level of miR-216b. The qPCR results revealed that miR-216b was increased markedly by introducing miR-216b mimics (Fig. 1E and $\mathrm{F}, \mathrm{p}<0.001)$. But miR-216b inhibitors did not exert its function on the endogenous miR$216 \mathrm{~b}$ (Data not shown). It might be the cause of the too low expression of miR-216b in ccRCC cells.

\section{MiR-216b directly targets and inhibits KRAS}

To explore the function of miR-216b, three computational algorithms: TargetScan, PicTar and miRanda, were used to search for potential miR-216b target genes and a large number of different target genes were predicted. In addition, in our preliminary next sequencing results [16], six genes (KRAS, BCL11B, CSK, RP2, ZFC3H1 and RPGRIP1L) were analyzed as targets of miR-216b. Combining these two sets of data, KRAS attracted our attention immediately. KRAS was a master regulator of cell growth, proliferation, differentiation and carcinogenesis. We detected KRAS protein level in ten pairs of clinical clear cell renal cell carcinoma specimens and adjacent specimens. It was observed that KRAS was dramatically upregulated in six ccRCC tissues (Fig. 1D). In addition, a miR-216b-binding site was found in the 3'-UTR of KRAS mRNA. There was perfect base pairing between the seed sequence

Fig. 2. KRAS is a direct target of miR-216b. (A) Sequence alignment of miR-216b and KRAS 3'-UTR. The KRAS 3'UTR contains one predicted miR-216b-binding site. The seed regions of miR-216b and the seed-recognizing sites in the KRAS 3'-UTR are indicated in red. (B) Luciferase assay on 786-0 cells, which were cotransfected with miR-216b and a luciferase reporter containing the sequence of KRAS 3'-UTR $\left(\mathrm{pSi}-\mathrm{KRAS}_{\mathrm{wt}}\right)$ or a mutant $(\mathrm{pSi}$ KRAS $_{\text {mut }}$ ) in which the ten nucleotides of the miR-216bbinding site were mutated. An empty luciferase reporter construct was used as a negative control (pSi-Ctrl). Luciferase activities were measured 48 hours post transfection. MiR216b markedly suppressed

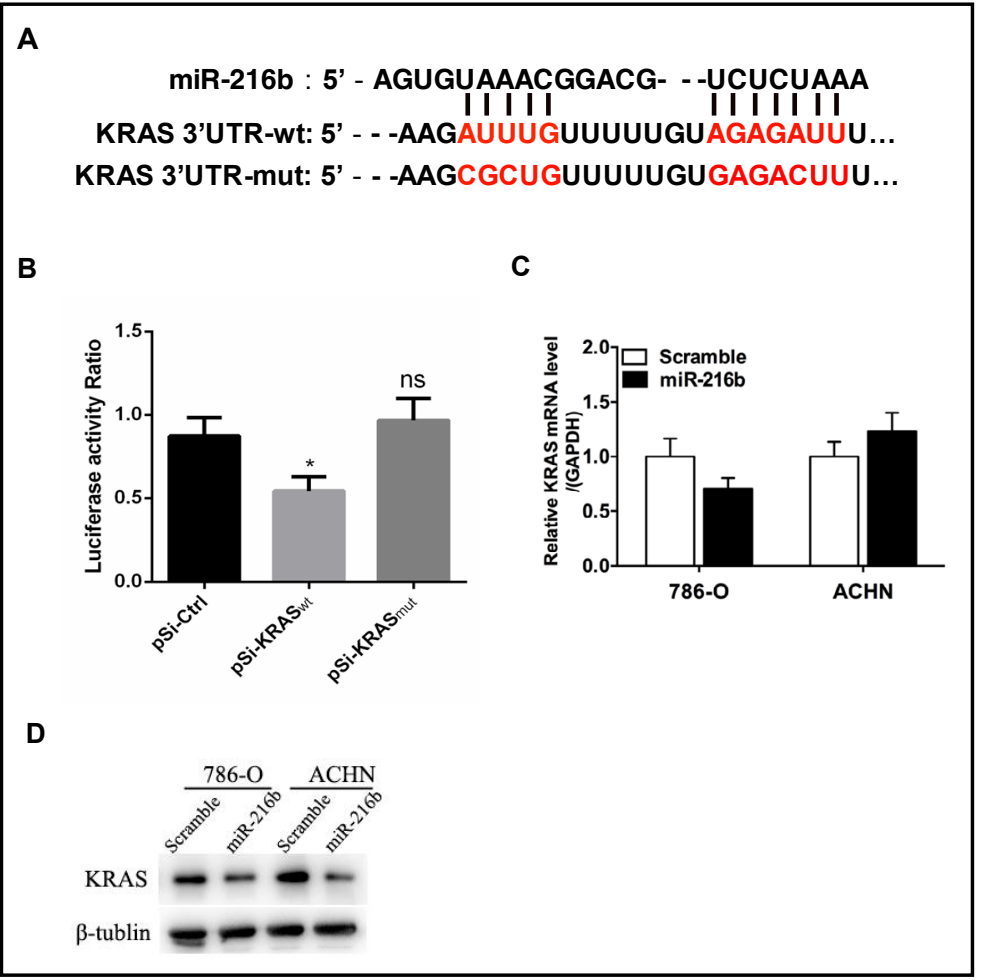
luciferase activity in pSi-KRAS reporter constructs. (C) Efficacy of miR-216b on KRAS mRNA level in ccRCC cells. The qPCR results revealed that KRAS mRNA level was not decreased by miR-216b in 786-0 and ACHN cells. (D) Efficacy of miR-216b on KRAS protein expression level in ccRCC cells. Western blotting results showed that KRAS protein expression was markedly decreased because of restore of miR-216b in 786-0 and ACHN cells. Mean \pm SEM, t-test, ${ }^{*} \mathrm{p}<0.05$.

\section{KARGER}


of mature miR-216b and the 3'-UTR of KRAS mRNA (Fig. 2A). To verify whether KRAS is a direct target of miR-216b, we subcloned the sequences of KRAS 3'-UTR into a luciferase reporter vector pSicheck- 2 . It showed that addition of miR-216b dramatically suppressed the luciferase activity of the KRAS 3'-UTR upon co-transfection of the luciferase vector (wildtype or mutant) with miR-216b into ccRCC cells (Fig. 2B, p<0.05). To directly assess the effect of miR-216b on KRAS expression, we transfected miR-216b into 786-O or ACHN cells and found that overexpression of miR-216b reduced KRAS protein levels (Fig. 2D). Much to our surprise, overexpression of miR-216b did not affect KRAS mRNA levels (Fig. 2C) although

Fig. 3. MiR216b inhibits cell proliferation and invasion. (A-D) Overexpression of miR-216b arrested cell proliferation at $48 \mathrm{~h}$ and $72 \mathrm{~h}$ after transfection with miR-216b mimics. But this was rescued upon coexpression of exogenous KRAS in 786-O and ACHN cells. Cells were plated in 96-well plates at the desired cell concentrations after transfection and cell viability was determined by CCK-8 assay at $0,24,48$ and 72 hours. (E-H) miR216b overexpression decreased cell invasion in 786-O and ACHN cells. Cells were transfected with miR216b mimics (or scramble) together with KRAS (or mock) transfection. All cells were subjected to a matrigel invasion assay. The overexpression of miR-216b inhibited the cell invasive potentials of 786-O and ACHN cells, whereas reexpression of KRAS rescued this inhibition. All data are shown as Means \pm SEM, ${ }^{*} \mathrm{p}<0.05$, ${ }^{* *} \mathrm{p}<0.01,{ }^{* * *} \mathrm{p}<0.001$.
A

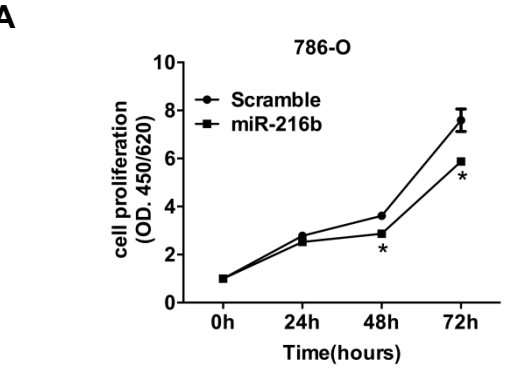

C

$786-0$

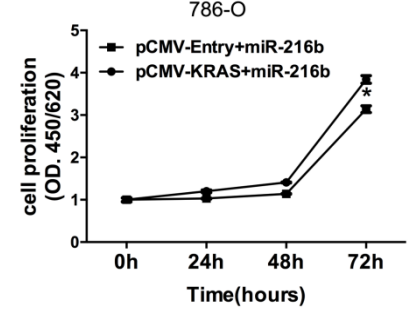

$E$

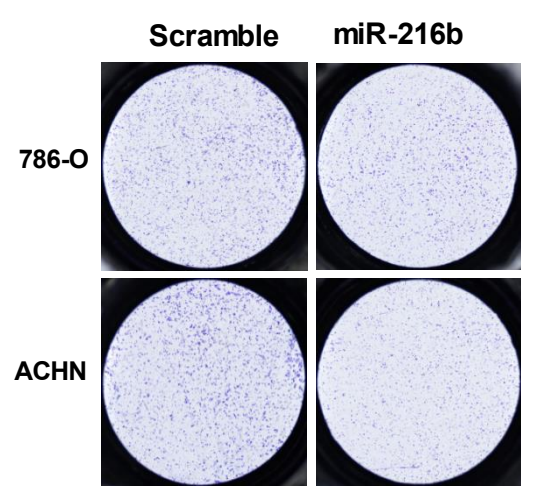

G

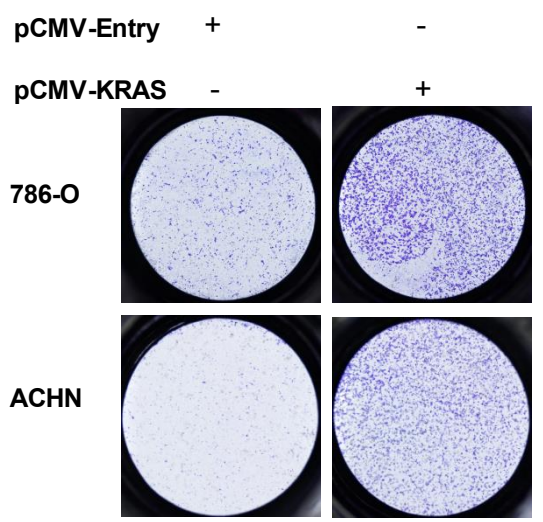

B

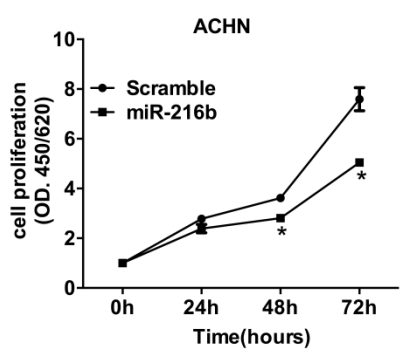

D

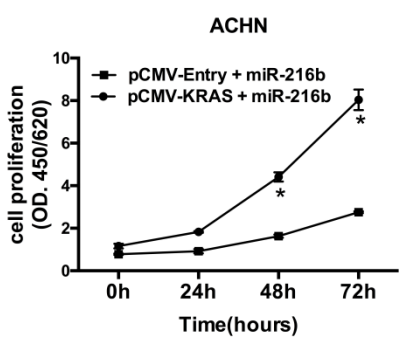

$\mathbf{F}$
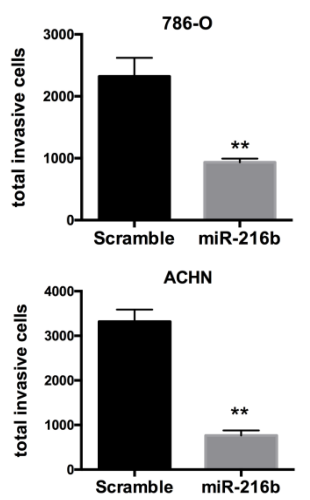

H

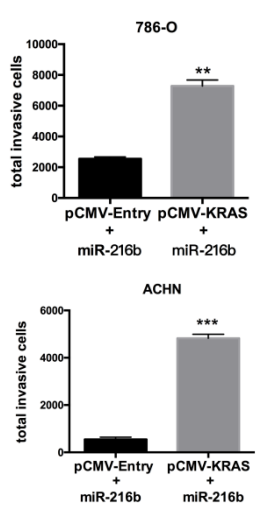


intracellular levels of miR-216b were altered significantly after treatment with miR-216b mimics (Fig. 1E and $\mathrm{F}, \mathrm{p}<0.001$ ). These results provide evidence that miR-216b directly binds to the 3'-UTR of KRAS mRNA and inhibits KRAS protein translation.

\section{MiR-216b suppresses cell proliferation and invasion by targeting KRAS}

In light of the above findings, we decided to explore the biological significance of miR$216 \mathrm{~b}$ in ccRCC tumorigenesis. We found that overexpression of miR-216b in 786-0 and ACHN cells markedly attenuated cell proliferation (Fig. 3A and B) and that the restoration of KRAS (without an endogenous 3'-UTR) rescued this inhibition (Fig. 3C and D), suggesting that miR-216b targets KRAS specifically. The overexpression of miR-216b inhibited the cell invasive potentials of 786-O and ACHN cells (Fig. 3E and F), whereas re-expression of KRAS rescued this inhibition (Fig. 3G and H). Our findings demonstrate that miR-216b has properties consistent with tumor suppressor function. The ability to modulate KRAS protein levels might explain, at least in part, why miR-216b can inhibit cell proliferation and invasion in ccRCC.

Next we used the subcutaneous xenograft mouse models to assess the impact of miR$216 \mathrm{~b}$ in tumor growth in vivo. The ACHN cells with agomir-216b or negative scramble control were subcutaneously injected into the flanks of balb/c nu-nu mice, and the tumor volumes were measured twice a week. At 42 days after implantation, the mice were sacrificed. The agomir-216b group exhibited a significant reduction in both tumor volume and weight compared with those of the negative control group (Fig. 4A-C). These data suggest that miR$216 \mathrm{~b}$ inhibits tumor growth of ccRCC in vivo.

\section{MiR-216b inhibits the AKT and ERKs pathways}

KRAS activation can trigger several important signaling pathways, such as the PI3K/ AKT and MAPK/ERK pathways, most of which regulate cell proliferation and invasion [1720]. Therefore, we investigated the possibility that miR-216b regulates those pathways by targeting KRAS. Restore of miR-216b, through transfection of miR-216b mimics, in 786-O and ACHN cells decreased the phosphorylation levels of AKT (Fig. 5A). Similarly, miR-216b suppressed the levels of phosphorylated ERK (Fig. 5A). These western blotting results demonstrate that miR-216b is a negative regulator of AKT and ERK pathways. Subsequently, rescue experiments were performed by overexpressing the KRAS vector (without an endogenous 3'- UTR) in miR-216b-treated cells: 786-O or ACHN cells were

Fig. 4. MiR-216b inhibits tumorigenesis in vivo. (A)The effect of miR-216b on in vivo tumorigenesis was analyzed by a subcutaneous tumor growth assay. ACHN cells with agomir$216 \mathrm{~b}$ or negative scramble control were subcutaneously injected into the flanks of balb/c nu-nu mice, respectively. Tumor volume was estimated at the indicated time points $\left({ }^{*} \mathrm{p}<0.05\right.$; mean \pm SEM; $n=6$ per group). (B) Mice were sacrificed, and the weight of subcutaneous tumors was measured at the end of the experiment $\left({ }^{*} \mathrm{p}<0.05\right.$; mean $\pm \mathrm{SEM}$; $n=6$ per group). (C) Final tumor photographs at 42 days postsubcutaneous injection.

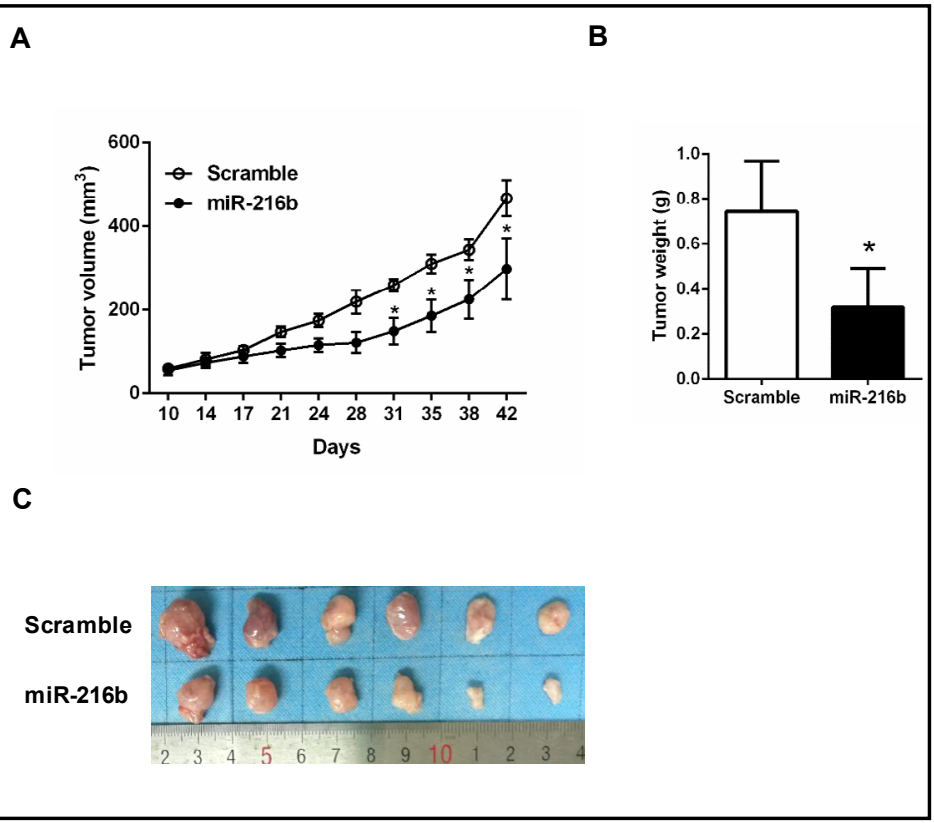


Fig. 5. MiR-216b inhibits AKT and ERK pathways by targeting KRAS. (A) Restoration of miR-216b reduced the activity of AKT and ERK pathways in 786-O and ACHN cells. (B) miR-216b transfection together with KRAS or mock vector in 786-0 and ACHN cells affects AKT and ERK signaling. AKT pathway activity was measured by examining expression of phosphorylated AKT (p-AKT), whereas EKR pathway activity was measured by examining expression of phosphorylated ERK (p-ERK1/2).
A

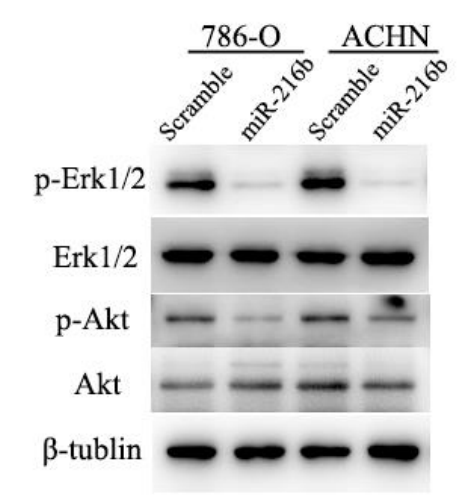

B

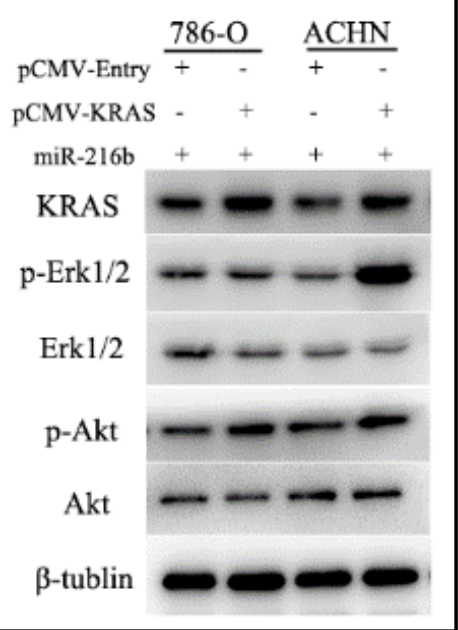

co-transfected with miR-216b mimics and KRAS-encoding vector (pCMV-KRAS). The miR$216 \mathrm{~b}$-induced downregulation of KRAS was rescued upon the introduction of KRAS, and the phosphorylation levels of AKT and ERK were altered in a similar manner (Fig. 5B). The downregulation of phosphorylated AKT and phosphorylated ERK by miR-216b could be rescued by re-expression of KRAS (Fig. 5B). These observations suggest that miR-216b inhibits the AKT and ERK pathways by targeting KRAS.

\section{Discussion}

Until now, only a handful of studies have identified specific miRNAs involved in human ccRCC tumorigenesis and metastasis. Therefore, we believe more effort should be made, not only towards the identification of relevant miRNAs but also into the specific mechanisms by which they accomplish their specific functions, particularly with regard to the oncogenesis of different types of tumors. Ding et al. found that miR-367 expression was increased in both human ccRCC tissues and cell lines and that ectopic high expression of miR-367 could promote cell proliferation and metastasis by targeting metastasis associated protein 3 (MTA3) in ccRCC [21]. Yoshino $\mathrm{H}$ et al. reported that miR-210-3p was downregulated in ccRCC, and the deletion of miR-210-3p promoted tumorigenesis through revival of TWIST1 [22].

In a preliminary study, we found miR-216b was downregulated in 10 ccRCC tissues compared with the matched adjacent normal tissues by miRNA sequencing [16]. Here, we used qRT-PCR to assess the level of miR-216b in ccRCC cell lines and 31 clinical specimens and found that miR-216b was indeed downregulated in ccRCC (Fig. 1A and B). In addition, we have shown that ectopic expression of miR-216b is associated with ccRCC cell growth and metastasis (Fig. 3A-H). Furthermore, we explored the potential targets of miR-216b in ccRCC cells via three miRNA target predict tools, and concerned the preliminary data also. It revealed KRAS as a candidate target of miR-216b, which attracted our attention immediately. Like other members of the RAS family, KRAS is a GTPase and an early player in many signal transduction pathways. Active GTP-bound KRAS associates with a wide variety of effectors, including Raf, PI3K, Ral-GDS, Rho GTPases and other molecules, to transmit downstream signals controlling distinct cellular events, including cell proliferation, survival, differentiation and invasion [17, 23]. Interestingly, KRAS gene mutations are rare in breast 


\section{Cellular Physiology Cell Physiol Biochem 2018;49:1755-1765

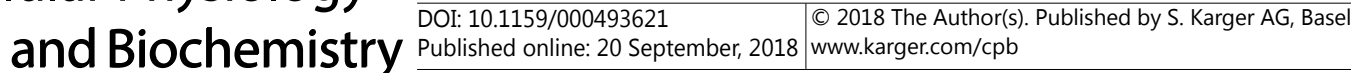 \\ Wang et al.: miR-216b, a Tumor Suppressor in ccRCC}

cancer [24] and nasopharyngeal carcinoma [25], but its upregulation has been found in these tumors analyzed [26-29]. The same situation has been observed in renal cell carcinomas. Hence, KRAS mutations do not appear to play a major role in these types of tumors [13], but several studies have revealed that KRAS overexpression is a frequent event in these tumors [14]. Here, restoration of miR-216b inhibits KRAS protein level in ccRCC cells (Fig. 2D). Moreover, the luciferase activity assays demonstrated that miR-216b could bind to the 3'UTR of KRAS mRNA (Fig. 2B). All of these data suggest that miR-216b directly targets KRAS expression in ccRCC cells. MiRNAs regulate gene expression through suppressing translation or inducing degradation of the target mRNAs. In the current study, we also investigated the effect of miR-216b on KRAS mRNA levels and found that miR-216b did not modulate KRAS mRNA levels (Fig. 2C). This implies that miR-216b targets KRAS through inhibiting translation, not by degrading mRNA.

KRAS has been shown to be activated in various tumor types. It is well known that active RAS transduces signals from cell surface receptors into the cytoplasm through specific effector pathways and regulates diverse cellular processes. The classic pathways downstream of KRAS are the MAPK/ERK and PI3K/AKT pathways $[17,19]$. The ability to target KRAS transcripts signifies that miR-216b might be a potential regulator of the MEK/ ERK and PI3K/AKT pathways in ccRCC cells. Our results have demonstrated that miR-216b can inhibit the constitutive activity of AKT and ERK pathways through targeting KRAS (Fig. $5 A$ ), whereas expression of exogenous KRAS (without an endogenous 3'-UTR) can rescue this inhibition (Fig. 5B). Given that KRAS plays a significant role in the regulation of cell proliferation and invasion, we investigated the effect of miR-216b on these phenotypes of ccRCC cells. MiR-216b overexpression in 786-0 and ACHN cells markedly attenuated the ability of the cells to proliferate and invade (Fig. 3A, B, E and F, Fig. 4 A-C), whereas overexpression of KRAS (without an endogenous 3'-UTR) rescued the inhibition of cell growth and the invasion caused by miR-216b, suggesting target specificity (Fig. 3C, D, G and $\mathrm{H}$ ). KRAS is an upstream modulator of many pathways, including AKT and ERK, through which it controls cell proliferation and invasion. Our findings suggest that miR-216b functions as a tumor suppressor, affecting ccRCC cell proliferation and invasion by targeting KRAS and subsequently suppressing downstream AKT and ERK signaling pathways. However, to completely clarify the function of miR-216b in tumorigenesis, more candidate targets will have to be detected in more types of cancers.

\section{Acknowledgements}

This work was supported by the National Natural Science Foundation of China (No. 81572513 and No. 81602229), the Science and Technology Development Fund Project of Shenzhen (No. JCYJ20160425094551377 and JCYJ20170307161640543) and China Postdoctoral Science Foundation (No.2016M592516).

\section{Disclosure Statement}

The authors declare no conflict of interests.

\section{References}

1 Ljungberg B, Campbell SC, Choi HY, Cho HY, Jacqmin D, Lee JE, Weikert S, Kiemeney LA: The epidemiology of renal cell carcinoma. Eur Urol 2011;60:615-621.

-2 Dijkhuizen T, Van Den Berg E, Van Den Berg A, Van De Veen A, Dam A, Faber H, Buys CH, Störkel S, De Jong B: Genetics as a diagnostic tool in sarcomatoid renal-cell cancer. Int J Cancer 1997;72:265-269. 


\section{Cellular Physiology Cell Physiol Biochem 2018;49:1755-1765 \begin{tabular}{ll|l} 
and Biochemistry Published online: 20 September, 2018 & $\begin{array}{l}\text { (c) } 2018 \text { The Author(s). Published by S. Karger AG, Basel } \\
\text { www.karger.com/cpb }\end{array}$ \\
\hline
\end{tabular}}

Wang et al.: miR-216b, a Tumor Suppressor in ccRCC

3 Xiao W, Lou N, Ruan H, Bao L, Xiong Z, Yuan C, Tong J, Xu G, Zhou Y, Qu Y, Hu W, Gao Y, Ru Z, Liu L, Xiao H, Chen K, Yang H, Zhang X: Mir-144-3p Promotes Cell Proliferation, Metastasis, Sunitinib Resistance in Clear Cell Renal Cell Carcinoma by Downregulating ARID1A. Cell Physiol Biochem 2017;43:2420-2433.

4 Petrozza V, Pastore AL, Palleschi G, Tito C, Porta N, Ricci S, Marigliano C, Costantini M, Simone G, Di Carlo A, Gallucci M, Carbone A, Fazi F: Secreted miR-210-3p as non-invasive biomarker in clear cell renal cell carcinoma. Oncotarget 2017;8:69551-69558.

$>5$ Lu J, Wei J-H, Feng Z-H, Chen Z-H, Wang Y-Q, Huang Y, Fang Y, Liang YP, Cen JJ, Pan YH, Liao B, Chen WF, Chen W, Luo JH: miR-106b-5p promotes renal cell carcinoma aggressiveness and stem-cell-like phenotype by activating Wnt/ $\beta$-catenin signalling. Oncotarget 2017;8:21461-21471.

-6 Li Y, Chen D, Li Y, Jin L, Liu J, Su Z, Qi Z, Shi M, Jiang Z, Gui Y, Yang S, Mao X, Lai Y: Identification of miR-130b as an oncogene in renal cell carcinoma. Mol Med Rep 2016;13:1902-1908.

7 Khella HWZ, Daniel N, Youssef L, Scorilas A, Nofech-Mozes R, Mirham L, Krylov SN, Liandeau E, Krizova A, Finelli A, Cheng Y, Yousef GM: miR-10b is a prognostic marker in clear cell renal cell carcinoma. J Clin Pathol 2017;70:854-859.

-8 Jana S, Sengupta S, Biswas S, Chatterjee A, Roy H, Bhattacharyya A: miR-216b suppresses breast cancer growth and metastasis by targeting SDCBP. Biochem Biophys Res Commun 2017;482:126-133.

-9 Liu Y, Niu Z, Lin X, Tian Y: MiR-216b increases cisplatin sensitivity in ovarian cancer cells by targeting PARP1. Cancer Gene Ther 2017;24:208-214.

10 You Y, Tan J, Gong Y, Dai H, Chen H, Xu X, Yang A, Zhang Y, Bie P: MicroRNA-216b-5p Functions as a Tumorsuppressive RNA by Targeting TPT1 in Pancreatic Cancer Cells. J Cancer 2017;8:2854-2865.

11 Yuan P, Cao W, Zang Q, Li G, Guo X, Fan J: The HIF-2 $\alpha$-MALAT1-miR-216b axis regulates multi-drug resistance of hepatocellular carcinoma cells via modulating autophagy. Biochem Biophys Res Commun 2016;478:1067-1073.

12 He S, Liao B, Deng Y, Su C, Tuo J, Liu J, Yao S, Xu L: MiR-216b inhibits cell proliferation by targeting FOXM1 in cervical cancer cells and is associated with better prognosis. BMC Cancer 2017;17:673.

-13 Gattenlöhner S, Etschmann B, Riedmiller H, Müller-Hermelink H-K: Lack of KRAS and BRAF mutation in renal cell carcinoma. Eur Urol 2009;55:1490-1491.

14 Bayrak O, Sen H, Bulut E, Cengiz B, Karakok M, Erturhan S, Seckiner I: Evaluation of EGFR, KRAS and BRAF gene mutations in renal cell carcinoma. J Kidney Cancer VHL 2014;1:40-45.

15 Wang Y, Zhao Y, Herbst A, Kalinski T, Qin J, Wang X, Jiang Z, Benedix F, Franke S, Wartman T, Camaj P, Halangk W, Kolligs FT, Jauch KW, Nelson PJ, Bruns CJ: miR-221 Mediates Chemoresistance of Esophageal Adenocarcinoma by Direct Targeting of DKK2 Expression. Ann Surg 2016;264:804-814.

16 Li X, Chen J, Hu X, Huang Y, Li Z, Zhou L, Tian Z, Ma H, Wu Z, Chen M, Han Z, Peng Z, Zhao X, Liang C, Wang Y, Sun L, Chen J, Zhao J, Jiang B, Yang H, Gui Y, Cai Z, Zhang X: Comparative mRNA and microRNA expression profiling of three genitourinary cancers reveals common hallmarks and cancer-specific molecular events. PLoS ONE 2011;6:e22570.

17 Campbell PM, Groehler AL, Lee KM, Ouellette MM, Khazak V, Der CJ: K-Ras Promotes Growth Transformation and Invasion of Immortalized Human Pancreatic Cells by Raf and Phosphatidylinositol 3-Kinase Signaling. Cancer Res 2007;67:2098-2106.

-18 Polosukhina D, Love HD, Correa H, Su Z, Dahlman KB, Pao W, Moses HL, Arteaga CL, Lovvorn HN, Zent R, Clark PE: Functional KRAS mutations and a potential role for PI3K/AKT activation in Wilms tumors. Mol Oncol 2017;11:405-421.

19 Calvo F, Agudo-Ibáñez L, Crespo P: The Ras-ERK pathway: Understanding site-specific signaling provides hope of new anti-tumor therapies. Bioessays 2010;32:412-421.

20 Kozma L, Kiss I, Nagy A, Szakáll S, Ember I: Investigation of c-myc and K-ras amplification in renal clear cell adenocarcinoma. Cancer Letters 1997;111:127-131.

21 Ding D, Zhang Y, Wen L, Fu J, Bai X, Fan Y, Lin Y, Dai H, Li Q Zhang Y, An R: MiR-367 regulates cell proliferation and metastasis by targeting metastasis-associated protein 3 (MTA3) in clear-cell renal cell carcinoma. Oncotarget 2017;8:63084-63095.

-22 Yoshino H, Yonemori M, Miyamoto K, Tatarano S, Kofuji S, Nohata N, Nakagawa M, Enokida H: microRNA210-3p depletion by CRISPR/Cas9 promoted tumorigenesis through revival of TWIST1 in renal cell carcinoma. Oncotarget 2017;8:20881-20894.

-23 Downward J: Targeting RAS signalling pathways in cancer therapy. Nat Rev Cancer 2003;3:11-22. 


\section{Cellular Physiology Cell Physiol Biochem 2018;49:1755-1765

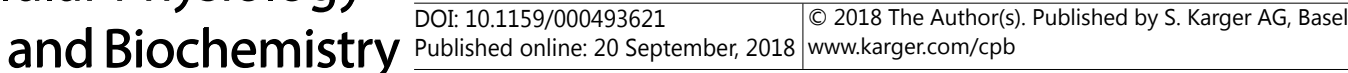

Wang et al.: miR-216b, a Tumor Suppressor in ccRCC

24 Myers MB, Banda M, McKim KL, Wang Y, Powell MJ, Parsons BL: Breast Cancer Heterogeneity Examined by High-Sensitivity Quantification of PIK3CA, KRAS, HRAS, and BRAF Mutations in Normal Breast and Ductal Carcinomas. Neoplasia 2016;18:253-263.

25 Deng M, Tang H, Zhou Y, Zhou M, Xiong W, Zheng Y, Ye Q, Zeng X, Liao Q Guo X, Li X, Ma J, Li G.: miR216b suppresses tumor growth and invasion by targeting KRAS in nasopharyngeal carcinoma. J Cell Sci 2011;124:2997-3005.

26 Thor A, Ohuchi N, Hand PH, Callahan R, Weeks MO, Theillet C, Lidereau R, Escot C, Page DL, Vilasi V: ras gene alterations and enhanced levels of ras p21 expression in a spectrum of benign and malignant human mammary tissues. Lab Invest 1986;55:603-615.

-27 Kiaris H, Spandidos DA, Jones AS, Vaughan ED, Field JK: Mutations, expression and genomic instability of the H-ras proto-oncogene in squamous cell carcinomas of the head and neck. Br J Cancer 1995;72:123-128.

-28 Yarbrough WG, Shores C, Witsell DL, Weissler MC, Fidler ME, Gilmer TM: ras mutations and expression in head and neck squamous cell carcinomas. Laryngoscope 1994;104:1337-1347.

29 Field JK: Oncogenes and tumour-suppressor genes in squamous cell carcinoma of the head and neck. Eur J Cancer, B, Oral Oncol 1992;28B:67-76. 